\title{
Acetylsalicylic acid use in primary prevention in Canada: Insight from the Primary Care Audit of Global Risk Management (PARADIGM) study
}

\author{
Milan Gupta MD FRCPC FACC ${ }^{1,2,3}$, Paul E Szmitko MD ${ }^{4}$, Mahesh Kajil MBBS CCRP1, Ilia Ostrovski BHSc ${ }^{1}$, \\ Michelle Tsigoulis Dip Nursing ${ }^{1}$, Subodh Verma MD PhD FRCSC FAHA ${ }^{3}$, Narendra Singh MD FRCPC FACC FAHA ${ }^{1,5}$
}

\begin{abstract}
M Gupta, PE Szmitko, M Kajil, et al. Acetylsalicylic acid use in primary prevention in Canada: Insight from the Primary Care Audit of Global Risk Management (PARADIGM) study. Curr Res Cardiol 2016;3(3):67-70.
\end{abstract}

BACKGROUND: Acetylsalicylic acid (ASA) is of proven benefit in reducing cardiovascular $(\mathrm{CV})$ events in both acute and chronic $\mathrm{CV}$ conditions. However, the role of ASA for the primary prevention of CV disease remains controversial, compounded by the risk of major hemorrhage with ASA therapy. Despite this, ASA remains frequently used in primary prevention.

METHODS: The PARADIGM study is an observational registry of 3015 healthy, middle-age Canadians, free of CV disease or diabetes, who underwent $\mathrm{CV}$ risk stratification. The present analysis assessed the use of ASA in this primary prevention cohort.

RESULTS: A total of 406 subjects (13.5\%) were prescribed ASA by their primary care physician. Those prescribed ASA, compared with

$\mathrm{T}$ he ability of acetylsalycylic acid (ASA) to irreversibly inactivate platelet cyclooxygenase and, hence, prevent the synthesis of thromboxane A2, which promotes vasoconstriction and platelet aggregation, makes it an essential component of the management of cardiovascular disease (CVD). The benefits of ASA therapy in patients with a history of CVD are well established. ASA use in the secondary prevention of CVD results in an approximately 20\% relative risk reduction (RRR) in CVD events including myocardial infarction and ischemic stroke, and approximately 10\% RRR in CVD mortality and total mortality compared with placebo (1). In the secondary prevention population, the associated increase in gastrointestinal and intracranial bleeding associated with ASA use is offset by the absolute benefit obtained from a reduction in cardiovascular events. However, the role for routine ASA use in the primary prevention of CVD is less clear.

The most recent guidelines from the American College of Chest Physicians (ACCP [2]), American Heart Association (AHA [3]), US Preventive Services Task Force (4) and European Society of Cardiology (ESC [5]) recommend the selective use of ASA in the primary prevention of CVD in older patients ( $>50$ years of age) and in those otherwise deemed to be at higher risk. Specifically, the ESC prevention guidelines indicate that in asymptomatic individuals, ASA use should only be considered when the 10 -year risk of CVD mortality is $>10 \%$ and blood pressure is controlled. Additionally, the Antithrombotic Trialists's Collaboration (ATTC) has indicated that in primary prevention without previous disease, ASA use is of uncertain net value as the

\begin{abstract}
those who were not, were more likely to be older, of male sex, white Caucasian, past/current smokers, hypertensive and to have a family history of premature CV disease. Blood pressure, renal function, high-sensitivity C-reactive protein, waist circumference and body mass index were all discriminators of ASA use. The mean modified Framingham risk score was significantly higher for those prescribed ASA compared with those who were not. Importantly, only $44 \%$ of those prescribed ASA were at high risk according to Framingham risk score.

CONCLUSIONS: The contemporary use of ASA in primary prevention remains high. Several clinical and laboratory factors influence the decision to prescribe ASA. However, the majority of ASA use was noted in individuals at low and intermediate risk (versus those at high risk).
\end{abstract}

Key Words: Acetylsalicylic acid; Cardiovascular disease; Primary prevention; risk stratification

reduction in occlusive events needs to be weighed against any increase in major bleeds $(1,6,7)$. Similarly, the Canadian Cardiovascular Society antiplatelet therapy recommendations for primary prevention do not recommend the routine use of ASA to prevent ischemic vascular events for either men or women without evidence of manifest vascular disease, unless the individual's vascular risk is considered to be high and bleeding risk is low (8). Thus, at present, it remains unclear whether the routine use of ASA in the primary prevention of CVD is warranted given the significant risks that are associated with its chronic use.

The Primary Care Audit of Global Risk Management (PARADIGM) study is a registry of 3015 patients who at baseline were free of CVD or diabetes. These subjects were enrolled by Canadian primary care physicians with the aim of evaluating risk stratification practices and use of both classic and novel cardiovascular risk markers in cardiac risk assessment. In the present analysis, we analyzed the frequency of ASA use in the primary prevention of CVD in this cohort and the factors influencing physician practice.

\section{METHODS}

The PARADIGM study is an observational registry that prospectively enrolled 3015 healthy, middle-age adults undergoing cardiovascular risk assessment across Canada between March, 2009 and February, 2010 by 105 primary care physicians. Nine eligibility criteria included: males ( $>40$ years of age) or females ( $>50$ years of age) undergoing CVD risk assessment and able and willing to provide informed

${ }^{1}$ Canadian Cardiovascular Research Network, Brampton; ${ }^{2}$ Department of Medicine, McMaster University, Hamilton; ${ }^{3}$ Division of Cardiac Surgery, Keenan Research Centre in the Li Ka Shing Knowledge Institute of St Michael's, University of Toronto; ${ }^{4}$ Division of Cardiology, Humber River Hospital and Sunnybrook Health Sciences Centre, Toronto, Ontario; ${ }^{5}$ Division of Cardiology, Medical College of Georgia at Augusta University, Augusta, Georgia, USA

Correspondence: Dr Milan Gupta, Canadian Cardiovascular Research Network, 3 Conestoga Drive, Suite 301, Brampton, Ontario L6Z 4 N5. Telephone 905-453-8885, fax 905-456-8885, e-mail mkgupta@rogers.com 


\begin{tabular}{|c|c|}
\hline \multicolumn{2}{|l|}{ Characteristics } \\
\hline Age, years & $56.3 \pm 8.4$ \\
\hline Male sex, \% & 58.8 \\
\hline Caucasian, \% & 69.6 \\
\hline Hypertension, \% & 30.4 \\
\hline Treated hypertension, \% & 26.6 \\
\hline Systolic blood pressure, $\mathrm{mmHg}$ & $126.6 \pm 14.0$ \\
\hline Diastolic blood pressure, $\mathrm{mmHg}$ & $78.5 \pm 9.1$ \\
\hline Past or current smoking, \% & 34.7 \\
\hline Family history of premature cardovascular disease, $\%$ & 24.3 \\
\hline Body mass index, $\mathrm{kg} / \mathrm{m}^{2}$ & $27.8 \pm 5.3$ \\
\hline Waist circumference, $\mathrm{cm}$ & $94.8 \pm 13.6$ \\
\hline \multicolumn{2}{|l|}{ Medications at baseline, $\mathrm{n}(\%)$} \\
\hline Acetylsalicylic acid & $406(13.5)$ \\
\hline Angiotensin-converting enzyme inhibitor & $327(10.9)$ \\
\hline Angiotensin receptor blocker & $333(11.0)$ \\
\hline Beta-blocker & $120(4.0)$ \\
\hline Calcium channel blocker & $166(5.5)$ \\
\hline Diuretic & $444(14.7)$ \\
\hline
\end{tabular}

Continuous variables are expressed as mean $\pm S D$

consent. Exclusion criteria included previous history of atherosclerotic disease (angina, myocardial infarction, transient ischemic attack, stroke, peripheral arterial disease or revascularization), a known high $(\geq 20 \%)$ Framingham risk score (FRS) or diabetes. Patients who were currently or previously taking a lipid-lowering agent were also excluded from the study.

Assessment of risk factors and cardiovascular biomarkers

Each patient underwent a standard cardiovascular assessment, which included a detailed history and physical examination. Smoking status, self-reported ethnicity and family history of CVD were recorded. At the initial visit, body weight, height and waist circumference were measured and baseline medications were reviewed. Blood pressure (BP) measurements were performed in accordance with Canadian Hypertension Education Program recommendations (10). Laboratory investigations including a fasting lipid profile, fasting glucose, glycated hemoglobin (HbA1c), serum creatinine (to estimate glomerular filtration rate $[\mathrm{eGFR}]$ ) and high-sensitivity C-reative protein (hsCRP) levels were performed by the primary care physician's local laboratory. The 10-year global cardiovascular risk score was calculated centrally for each patient using the FRS (ATP-III) (11). Individuals were assigned to either very low $(<5 \%)$, low ( $5 \%$ to $9 \%$ ), intermediate (10-\% to $19 \%$ ) or high (>20\%) 10-year risk categories.

\section{Statistical methods}

Continuous variables are expressed as mean \pm SD. Welch's $t$ test, assuming unequal variances, was used to test the difference in means for continuous variables. The Fisher's exact test was used to test the difference in proportions from two independent samples for categorical variables. Categorical variables are presented as counts and percentages. Logistic regression was used for the multivariate analysis. Variables that appeared to be significant at $5 \%$ in the univariate analysis were selected in the multivariate model. All statistical tests were two-tailed, and $\mathrm{P}<0.05$ was considered to be statistically significant.

\section{RESULTS}

The clinical characteristics of the entire PARADIGM cohort are presented in Table 1. The mean ( \pm SD) age of the cohort was $56.3 \pm 8.4$ years; $58.8 \%$ were male, $69.6 \%$ were Caucasian, $34.7 \%$ had a history of past/current smoking, $30.4 \%$ had a history of hypertension and $24.3 \%$
TABLE 2

Cardiovascular risk factors and overall Framingham risk scores in acetylsalicylic acid (ASA) and non-ASA users

\begin{tabular}{|c|c|c|c|}
\hline \multirow[b]{2}{*}{ Characteristic } & \multicolumn{2}{|c|}{ ASA } & \multirow[b]{2}{*}{$\mathbf{P}$} \\
\hline & $\begin{array}{l}\text { Users } \\
(n=406)\end{array}$ & $\begin{array}{c}\text { Nonusers } \\
(n=2609)\end{array}$ & \\
\hline Age, years & $61.6 \pm 8.5$ & $55.5 \pm 8.1$ & $<0.0001$ \\
\hline Male sex & $255(62.8)$ & $1519(58.2)$ & $<0.001$ \\
\hline Caucasian & 361 (88.9) & $1738(66.6)$ & $<0.00001$ \\
\hline Hypertension & $247(60.8)$ & $670(25.7)$ & $<0.00001$ \\
\hline Past/current smoking & $178(43.8)$ & $868(33.3)$ & $<0.001$ \\
\hline Family history of CVD & $125(30.9)$ & $607(23.3)$ & 0.002 \\
\hline $\mathrm{BMI}\left(>25 \mathrm{~kg} / \mathrm{m}^{2}\right)$ & $314(77.3)$ & $1764(67.6)$ & $<0.0005$ \\
\hline $\mathrm{BMI}\left(>30 \mathrm{~kg} / \mathrm{m}^{2}\right)$ & $140(34.5)$ & $685(26.3)$ & $<0.0005$ \\
\hline LDL-c, mmol/L & $3.55 \pm 0.83$ & $3.57 \pm 0.84$ & 0.77 \\
\hline Fasting glucose, $\mathrm{mmol} / \mathrm{L}$ & $5.4 \pm 0.7$ & $5.4 \pm 0.7$ & 0.16 \\
\hline $\mathrm{IFG}(>6.1 \mathrm{mmol} / \mathrm{L})$ & $45(11.1)$ & $236(9.0)$ & 0.20 \\
\hline $\mathrm{HbA} 1 \mathrm{c}, \%$ & $5.7 \pm 0.4$ & $5.7 \pm 0.4$ & 0.54 \\
\hline eGFR, $\mathrm{mL} / \mathrm{min} / 1.73 \mathrm{~m}^{2}$ & $72.7 \pm 16.5$ & $78.1 \pm 15.7$ & $<0.0005$ \\
\hline hsCRP (>2.0 mg/L) & $200(49.3)$ & $1052(40.3)$ & $<0.0005$ \\
\hline Very low FRS (<5\%), \% & 2.2 & 15.0 & $<0.00001$ \\
\hline Low FRS $(5 \%-9 \%), \%$ & 15.8 & 32.6 & $<0.00001$ \\
\hline $\begin{array}{l}\text { Intermediate FRS (10\% - } \\
19 \%), \%\end{array}$ & 37.7 & 33.3 & $<0.00001$ \\
\hline High FRS ( $\geq 20 \%), \%$ & 44.3 & 19.1 & $<0.00001$ \\
\hline Mean FRS, \% & $22.3 \pm 14.0$ & $13.5 \pm 10.3$ & $<0.00001$ \\
\hline
\end{tabular}

Data presented as $n$ (\%) unless otherwise indicated, continuous variables are expressed as mean $\pm S D$. BMI Body mass index; CVD Cardiovascular disease; eGFR Estimated glomerular filtration rate; $H b A 1 c$ Glycated hemoglobin; hsCRP High-sensitivity C-reactive protein; IFG Impaired fasting glucose; FRS Framingham risk score; LDL-c Low-density lipoprotein cholesterol

reported a family history of CVD. Of these 3015 participants, 406 (13.5\%) were prescribed ASA for primary CVD prevention by their family physicians. Table 2 shows the general characteristics of the subjects who were prescribed ASA for primary prevention. Significant differences were noted between subjects who were prescribed ASA and those who were not. When compared with their counterparts, patients taking ASA were older $(61.6 \pm 8.4$ years versus $55.5 \pm 8.1$ years $(\mathrm{P}<0.0001)$, more often male $(\mathrm{P}<0.001)$, more often Caucasian ( $88.9 \%$ versus $66.6 \% ; \mathrm{P}<0.0001)$, and more likely to be past or current smokers (43.8\% versus $33.3 \%$; $\mathrm{P}<0.001$ ), hypertensive ( $60.8 \%$ versus $25.7 \% ; \mathrm{P}<0.00001)$ and to have a family history of premature CVD $(30.9 \%$ versus $23.3 \% ; \mathrm{P}<0.01)$ (Table 2$)$. Systolic and diastolic BP, serum creatinine, hsCRP levels, waist circumference (WC) and body mass index (BMI) were also significantly higher among ASA versus non-ASA users (all $\mathrm{P}<0.0005)$ (Table 2$)$. However, low-density lipoprotein cholesterol (LDL-c) level, fasting blood glucose and HbA1c did not differ between the two groups (Table 2). In multivariate analysis, older age, male sex, the presence of hypertension, smoking, family history of CVD and an elevated BMI were independently associated with the use of ASA (Table 3). Individuals with a history of hypertension were 3.87 times more likely than nonhypertensive patients to be prescribed ASA (95\% CI 3.38 to 5.43 ).

The use of aspirin was evaluated as a function of the calculated FRS. Individuals were assigned to either very low $(<5 \%)$, low $(5 \%$ to $10 \%)$, intermediate (10\% to $20 \%$ ) or high (>20\%) 10 -year risk categories. The mean modified FRS for those prescribed ASA was higher $(22.3 \pm 14.0 \%$ ASA users versus $13.5 \pm 10.3 \%$ nonusers; $\mathrm{P}<0.00001)$ (Table 2). However, $44.3 \%$ of ASA users were in the high-risk category compared with $2.2 \%, 15.8 \%$ and $37.7 \%$ in the very low, low and intermediate risk groups, respectively (Table 2).

Stratification of the ASA-treated patients based on sex revealed no differences in the proportion of ASA-treated subjects, rates of smoking, presence of hypertension, presence of a family history of 
TABLE 3

Risk factors independently associated with acetylsalicylic acid use following multivariate analysis

\begin{tabular}{lcr}
\hline Characteristic & OR (95\% CI) & \multicolumn{1}{c}{$\mathbf{P}$} \\
\hline Age & $1.08(1.06-1.09)$ & $<0.00001$ \\
Male sex & $1.63(1.28-2.08)$ & 0.00007 \\
Caucasian & $3.53(2.50-4.99)$ & $<0.00001$ \\
Hypertension & $3.87(3.06-4.91)$ & $<0.00001$ \\
Family history of cardiovascular disease & $1.44(1.12-1.87)$ & 0.00482 \\
Body mass index & $1.03(1.00-1.05)$ & 0.02407 \\
\hline
\end{tabular}

CVD, BMI and hsCRP between men and women (Table 4). However, the mean FRS was lower in ASA-treated women than their male counterparts $\quad(14.4 \pm 8.0 \%$ versus $27.0 \pm 14.8 \% ; \quad \mathrm{P}<0.00001)$. Significantly more ASA-treated women than men were in the low $(34.4 \%$ versus $8.2 \% ; \mathrm{P}<0.00001)$ and intermediate $(43.0 \%$ versus $34.5 \%$; $<<0.00001)$ FRS categories, and significantly more men were in the high FRS than women $(57.3 \%$ versus $22.5 \%$; $\mathrm{P}<0.00001)$ (Table 4).

\section{DISCUSSION}

Although the value of ASA in the secondary prevention of cardiovascular events is widely accepted and endorsed by various national and international guidelines, the use of ASA in the primary prevention of cardiovascular events remains highly disputed $(1,6,12-14)$. The American College of Chest Physicians provides a 2B recommendation for the use of ASA in the primary prevention of asymptomatic individuals $>50$ years of age. However, the AHA suggests that the use of ASA for cardiovascular prophylaxis is recommended only for patients whose risk is sufficiently high for the benefits to outweigh the risks associated with the treatment (a 10-year risk of $6 \%$ to $10 \%$ ) (class 1 recommendation), which is similar to the recommendations of the ATTC and ESC respectively (1-3,5). The Canadian Cardiovascular Society antiplatelet therapy recommendations for primary prevention do not recommend the routine use of ASA to prevent ischemic vascular events for men or women without evidence of manifest vascular disease; however, they recommend that (8):

... in special circumstances in men and women without evi-

dence of manifest vascular disease in whom vascular risk is considered high and bleeding risk low, ASA 75-162 mg daily may be considered.

Similarly, the US Preventive Services Task Force encourages physicians to participate in shared decision making with patients and to recommend ASA use for the prevention of CVD when its potential CVD benefit (myocardial infarction in men and stroke in women) outweighs the potential harm of gastrointestinal hemorrhage.

Raju and Eikelboom (6) recently reviewed and summarized the results of the four large meta-analyses addressing this issue. When used in primary prevention, ASA produces a nominally significant $6 \%$ reduction in all-cause mortality. Although ASA does not reduce cardiovascular mortality or cancer mortality, the pooled estimates for both of these outcomes are in favour of ASA, explaining the reduction in all-cause death. Furthermore, ASA use in primary prevention reduces the composite of major cardiovascular events by $10 \%$ to $13 \%$, reduces nonfatal myocardial infarction by $19 \%$ to $23 \%$, and ischemic stroke by $14 \%$. However, ASA use in primary prevention is associated with a $32 \%$ to $36 \%$ increased risk for hemorrhagic stroke, a $31 \%$ to $66 \%$ increase in major bleeding and a $37 \%$ increase in gastrointestinal bleeding. From the perspective of net benefit, 314 to 384 individuals would have to take ASA for an average of 6.9 years to prevent one major cardiovascular event, at the cost of about three gastrointestinal or major bleeds.

In the ATTC meta-analysis, the estimated absolute benefit of ASA in primary prevention in individuals in the very low risk category (global 10 -year risk $<5 \%$ ) is estimated to be $0.2 \%$, with a $0.1 \%$
TABLE 4

Differences in acetylsalicylic acid-treated subjects according to sex

\begin{tabular}{lccc}
\hline Characteristic & Male $(\mathbf{n}=\mathbf{2 5 5})$ & Female $(\mathbf{n}=\mathbf{1 5 1})$ & $\mathbf{P}$ \\
\hline Age, years & $60.6 \pm 8.7$ & $63.2 \pm 7.8$ & 0.002 \\
HDL-c, mmol/L & $1.26 \pm 0.33$ & $1.55 \pm 0.43$ & $<0.00001$ \\
LDL-c, mmol/L & $3.42 \pm 0.77$ & 3.770 .88 & 0.0001 \\
HbA1c, \% & $5.6 \pm 0.4$ & $5.9 \pm 0.4$ & 0.0001 \\
Smoking history, \% & 46.7 & 39.1 & 0.15 \\
Hypertension, \% & 58.4 & 64.9 & 0.21 \\
Family history of CVD, \% & 30.7 & 31.1 & 1.0 \\
Body mass index, kg/m ${ }^{2}$ & $29.4 \pm 5.4$ & $28.5 \pm 6.4$ & 0.17 \\
hsCRP, mg/L & $3.5 \pm 5.9$ & $3.7 \pm 3.5$ & 0.67 \\
Low FRS (<10\%) & 8.2 & $34.4 \%$ & $<0.00001$ \\
Intermediate FRS (10\% to & 34.5 & $43.0 \%$ & $<0.00001$ \\
$\quad$ 19\%), \% & & & $<0.00001$ \\
High FRS ( $\geq 20 \%), \%$ & 57.3 & 22.5 & $<0.00001$ \\
Mean FRS, \% & $27.0 \pm 14.8$ & $14.4 \pm 8.0$ &
\end{tabular}

Continuous variables are expressed as mean \pm SD. BMI Body mass index; CVD Cardiovascular disease; FRS Framingham risk score; HbA1c Glycated hemoglobin; HDL-c High-density lipoprotein cholesterol; hsCRP Highsensitivity C-reactive protein; LDL-c Low-density lipoprotein cholesterol

increase in the risk of bleeding (1). However, in a higher-risk population, it is estimated that ASA use may be associated with an absolute $2 \%$ risk reduction with a $1 \%$ absolute bleeding risk, a calculation that would favour ASA use. Clinicians are, therefore, faced with the difficult task of trying to help identify appropriate candidates for ASA therapy wherein the risk reduction outweighs the potential bleeding risks.

Most recently, the Japanese Primary Prevention Project (JPPP) trial evaluated the use of low-dose ASA in $>14,000$ Japanese subjects $>60$ years of age who had concomitant risk factors for CVD (15). The trial was terminated for futility after a median follow-up of five years, with no benefit observed with ASA in major atherosclerotic events, yet a significant excess of major bleeding.

The Primary Care Audit of Global Risk Management (PARADIGM) study was established to evaluate primary care physicians' knowledge and attitudes towards global risk assessment and treatment in an otherwise healthy middle-age population of Canadians, free of cardiovascular disease or diabetes. Several important observations emanate from these data. First, the use of ASA in primary prevention is relatively modest at $13.5 \%$. Several clinical characteristics including advanced age, male sex, Caucasian ethnicity, hypertension, smoking, a family history of CVD, increased BMI or waist circumference, an elevated serum creatinine, an increased hsCRP level and a higher FRS were associated with ASA use in primary prevention in the univariate analysis. However, in the multivariate analysis, only age, male sex, Caucasian ethnicity, elevated BMI, hypertension and a family history of CVD were independent discriminators of ASA use.

When we examined the appropriateness of therapy by examining the distribution of ASA use among the various FRS subgroups, we noted an appropriate increase in ASA use in patients with higher CV risk. However, the majority of ASA use (55\%) occurred in patients at low or intermediate risk, groups in which the risk:benefit ratio of ASA remains uncertain. Although this trend was seen in men and women, the magnitude was greater in women, in that approximately $80 \%$ of ASA use was in the low- and intermediate-risk groups.

In summary, we provide a contemporary analysis of ASA use in the primary prevention of CVD in a large number of otherwise healthy middle-age Canadians free of CVD or diabetes. Our assessment reveals fairly frequent use of ASA in low- and intermediate-risk patients, more so in women than in men. Given the uncertainty of benefit of ASA in this population, further studies and clarification of guidelines for the use of ASA in primary prevention are required. 
ACKNOWLEDGMENTS: Farah Thong assisted with editing the manuscript and creating the reference database. This research was conducted with support from the Investigator-Sponsored Study Programme of AstraZeneca.

FUNDING: This study was funded by CCRN through an unrestricted investigator initiated research grant from AstraZeneca Canada Inc.

\section{REFERENCES}

1. Antithrombotic Trialists' (ATT) Collaboration, Baigent C, Blackwell L, et al. Aspirin in the primary and secondary prevention of vascular disease: collaborative meta-analysis of individual participant data from randomised trials. Lancet 2009;373:1849-60.

2. Vandvik PO, Lincoff AM, Gore JM, et al. Primary and secondary prevention of cardiovascular disease: Antithrombotic Therapy and Prevention of Thrombosis, 9th edn: American College of Chest Physicians Evidence-Based Clinical Practice Guidelines. Chest 2012;141:e637S-68S.

3. Goldstein LB, Bushnell CD, Adams RJ, et al. Guidelines for the primary prevention of stroke: A guideline for healthcare professionals from the American Heart Association/American Stroke Association. Stroke 2011;42:517-84.

4. Force USPST. Aspirin for the prevention of cardiovascular disease: U.S. Preventive Services Task Force recommendation statement. Ann Intern Med 2009;150:396-404.

5. Graham I, Atar D, Borch-Johnsen K, Boysen G, et al. European guidelines on cardiovascular disease prevention in clinical practice: Full text. Fourth Joint Task Force of the European Society of Cardiology and other societies on cardiovascular disease prevention in clinical practice (constituted by representatives of nine societies and by invited experts). Eur J Cardiovasc Prev Rehabil 2007;14(Suppl 2):S1-113.

6. Raju NC, Eikelboom JW. The aspirin controversy in primary prevention. Curr Opinion Cardiol 2012;27:499-507.

7. Barnett H, Burrill P, Iheanacho I. Don't use aspirin for primary prevention of cardiovascular disease. BMJ 2010;340:c1805.

8. Bell AD, Roussin A, Cartier R, et al. The use of antiplatelet therapy in the outpatient setting: Canadian Cardiovascular Society guidelines. Can J Cardiol 2011;27(Suppl A):S1-59.
DISCLOSURES: Milan Gupta has received speaking honoraria, consulting fees, and/or research grants from Bayer, Boehringer Ingelheim, BMS/ Pfizer and Lilly. Subodh Verma Has received speaking honoraria, consulting fees, and/or research grants from Abbott, AstraZeneca, Boehringer Ingelheim, Merck/Schering-Plough, Novartis, Pfizer and sanofi-aventis. Narendra Singh has received research grants and speakers bureau/honoraria for Jaansen, Bristol Myers Squibb, Boeringer Ingelheim and Daichi Sankyo. Mahesh Kajil, Ilia Ostrovski, Paul E Szmitko and Michelle Tsigoulis have no financial disclosures or conflicts of interest to declare.

9. Law T, Yan AT, Gupta A, Kajil M, Tsigoulis M, Singh N, Verma S, Gupta M. Primary prevention of cardiovascular disease: Global cardiovascular risk assessment and management in clinical practice (PARADIGM study). EHJ-quality of care and clinical outcomes, 31-36. Published online: June 2, 2015.

10. Campbell NR, Khan NA, Hill MD, et al. Canadian Hypertension Education Program recommendations: The scientific summary - an annual update. Can J Cardiol 2009;25:271-77.

11. Expert Panel on Detection E, Treatment of High Blood Cholesterol in A. Executive Summary of The Third Report of The National Cholesterol Education Program (NCEP) Expert Panel on Detection, Evaluation, And Treatment of High Blood Cholesterol In Adults (Adult Treatment Panel III). JAMA 2001;285:2486-97.

12. Bartolucci AA, Tendera M, Howard G. Meta-analysis of multiple primary prevention trials of cardiovascular events using aspirin. Am J Cardiol 2011;107:1796-801.

13. Raju N, Sobieraj-Teague M, Hirsh J, O’Donnell M, Eikelboom J. Effect of aspirin on mortality in the primary prevention of cardiovascular disease. Am J Med 2011;124:621-9.

14. Seshasai SR, Wijesuriya S, Sivakumaran R, et al. Effect of aspirin on vascular and nonvascular outcomes: Meta-analysis of randomized controlled trials. Arch Intern Med 2012;172:209-16.

15. Yasuo I, Kazuyuki S, Tamio T, et al. Low-dose aspirin for primary prevention of cardiovascular events in Japanese patients 60 years or older with atherosclerotic risk factors. JAMA 2014;312:2510-20. 